\title{
Intake of milk, but not total dairy, yogurt, or cheese, is negatively associated with the clustering of cardiometabolic risk factors in adolescents
}

Sandra Abreu, , , Pedro Moreira ${ }^{\mathrm{a}, \mathrm{b}}$, Carla Moreira ${ }^{\mathrm{a}}$, Jorge Motaa, Isabel Moreira-Silva ${ }^{\mathrm{a}}$, Paula-Clara Santos ${ }^{\mathrm{a}}$

c, Rute Santos a, d

${ }^{a}$ Research Centre in Physical Activity, Health and Leisure, Faculty of Sport, University of Porto, 91-4200 Porto, Portugal

${ }^{b}$ Faculty of Nutrition and Food Science, University of Porto, Porto, 91-4200 Porto, Portugal

Department of Physical Therapy, School of Health Technology of Porto, Polytechnic Institute of Porto, Vila Nova de Gaia, Portugal

d Maia Institute of Higher Education, Maia, Portugal

\begin{abstract}
Epidemiologic studies have reported an inverse association between dairy product consumption and cardiometabolic risk factors in adults, but this relation is relatively unexplored in adolescents. We hypothesized that a higher dairy product intake is associated with lower cardiometabolic risk factor clustering in adolescents. To test this hypothesis, a cross-sectional study was conducted with 494 adolescents aged 15 to 18 years from the Azorean Archipelago, Portugal. We measured fasting glucose, insulin, total cholesterol, high-density lipoprotein cholesterol, triglycerides, systolic blood pressure, body fat, and cardiorespiratory fitness. We also calculated homeostatic model assessment and total cholesterol/high-density lipoprotein cholesterol ratio. For each one of these variables, a $z$ score was computed using age and sex. A cardiometabolic risk score (CMRS) was constructed by summing up the $z$ scores of all individual risk factors. High risk was considered to exist when an individual had at least 1 SD from this score. Diet was evaluated using a food frequency questionnaire, and the intake of total dairy (included milk, yogurt, and cheese), milk, yogurt, and cheese was categorized as low (equal to or below the median of the total sample) or "appropriate" (above the median of the total sample). The association between dairy product intake and CMRS was evaluated using separate logistic regression, and the results were adjusted for confounders. Adolescents with high milk intake had lower CMRS, compared with those with low intake $(10.6 \%$ vs $18.1 \%, P=.018)$. Adolescents with appropriate milk intake were less likely to have high CMRS than those with low milk intake (odds ratio, 0.531; 95\% confidence interval, 0.302-0.931). No association was found between CMRS and total dairy, yogurt, and cheese intake. Only milk intake seems to be inversely related to CMRS in adolescents.
\end{abstract}
Abbreviations
BMI, body mass index;
BP, blood pressure;
CI, confidence interval; 
CMRS, cardiometabolic risk score;

CRF, cardiorespiratory fitness;

FFQ, food frequency questionnaire;

HDL-c, high-density lipoprotein cholesterol;

HOMA, homeostatic model assessment;

MetS, metabolic syndrome;

OR, odds ratio;

SES, socioeconomic status;

TC, total cholesterol;

TG, triglyceride

\section{Keywords}

Cardiometabolic risk;

Dairy product;

Milk;

Adolescents;

Cross-sectional study

\section{Introduction}

Cardiometabolic risk is characterized by a cluster of risk factors that includes obesity, abnormal glucose homeostasis, dyslipidemia, and hypertension [1] and [2]. In adults, the positive association between the clustering of cardiometabolic risk factors and cardiovascular disease and type 2 diabetes is clearly established, and a similar relationship has been described in children and adolescents [3] and [4]. Furthermore, it has been described that the clustering of cardiometabolic risk factors is a better measure of cardiovascular health in children than single-risk factors [5].

Irrespective of the impact of genetic factors, such modifiable lifestyle elements as dietary intake are generally considered to contribute to both the development and subsequent course of cardiometabolic risk [6]. In this context, scientific investigation has focused on understanding the role of diet in the development of cardiometabolic risk. It has been proposed that the intake of dairy products protects against cardiometabolic risk or several of its risk factors [1], [7] and [8]. Evidence, mostly in adults, has indicated that increased dairy intake may decrease the risk of high blood pressure (BP) [8] and [9], central obesity [10] and [11], and hyperinsulinemia [12]. It has been established that such dairy components as calcium, 
medium-chain fatty acids, and bioactive peptides may play an important role in the prevention of cardiometabolic risk and its complications via the exertion of mechanisms that include the satiety response and the regulation of insulinemia levels and BP [13].

Although evidence points out that dairy intake may help guard against cardiometabolic risk, gaps remain in the literature on the relationship between dairy intake and cardiometabolic risk in the adolescent population. However, previous studies have shown inverse associations between milk or dairy product intake and selected cardiometabolic risk factors [11], [14], [15] and [16]. In addition, questions remain as to whether different types of dairy products exert distinct effects against cardiometabolic risk. Hence, we have hypothesized that a higher dairy product intake is associated with lower cardiometabolic risk factors clustering in adolescents. In addition, the aim of this study was to identify the association between dairy product intake and the clustering of cardiometabolic risk factors in a sample of Portuguese adolescents.

\section{Methods and materials}

\subsection{Sampling}

Data for the present cross-sectional study came from a 2008 longitudinal school-based study—The Azorean Physical Activity and Health Study II-which aimed to evaluate physical activity, physical fitness, overweight/obesity prevalence, dietary intake, health-related quality of life, and other factors in 15- to 18year-old adolescents. This study was conducted in 6 of the 9 Azorean Islands (S. Miguel, Terceira, Faial, Pico, S. Jorge, and Graciosa), where 95\% of the Azorean population lives [17].

All participants in this study were informed of its goals, and the parent or guardian of each participant provided written informed consent for his/her child to participate. The study was approved by the Faculty of Sport, University of Porto, and the Portuguese Foundation for Science and Technology Ethics Committee; it was conducted in accordance with the World Medical Association's Helsinki Declaration for Human Studies.

The population was selected by means of a proportionate-stratified random sampling, taking into account the location (island) and the number of students, by age and sex, in each school. Baseline data were collected for 1515 adolescents in the fall of 2008 , and 850 subjects were reevaluated 1 year later (mean \pm SD followup length, $11.5 \pm 2.0$ months). In view of the fact that blood samples were only taken in 2009 , we just considered data from that year in this study. Of the 850 adolescents who participated in the 2009 data collection, 297 refused to undergo blood sampling, 36 were excluded because of lack of information on several variables, and 23 were excluded because of lack of information on dietary intake. Therefore, the final sample included in our cross-sectional analysis comprised 494 adolescents (208 boys). The subjects who were excluded from this study did not significantly differ from those who were included, with regard to age, parental education, and sex (data not shown). Finally, the sample was weighted in accordance with 
the distribution of the Azorean population in schools and so as to guarantee the real representativeness of each group (by age and sex).

\subsection{Anthropometric measures}

Body height and weight were determined using standard anthropometric methods [18]. Height was measured to the nearest millimeters in bare or stocking feet, with adolescents standing upright against a Holtain portable stadiometer (Crymych, Pembrokeshire, UK); weight was measured to the nearest $0.10 \mathrm{~kg}$, with participants lightly dressed (underwear and t-shirt) and with the use of a portable digital beam scale (Tanita Inner Scan BC 532, Tokyo, Japan).

Body mass index (BMI) was calculated using the ratio of weight/height ${ }^{2}$ (in kilograms per meter squared). Subjects were classified as normal weight, overweight, or obese, according to age- and sex-specific cutoff points that were specified by the International Obesity Task Force [19] and [20]. Underweight subjects (3.2\%) were combined with subjects in the normal-weight category, owing to the fact that they represented a small proportion of the sample. Percentage body fat was assessed using bioelectric impedance analysis (Tanita Inner Scan BC 532).

Waist circumference measurements were taken midway between the 10th rib and the iliac crest and recorded to $0.1 \mathrm{~cm}$. A nonelastic flexible tape measure was used, with subjects standing erect-arms by sides, feet together, and abdomen relaxed—as well as without clothing covering the waist area.

\subsection{Blood pressure}

Blood pressure was measured using Dynamap vital signs monitors, model BP 8800 (Critikon, Inc, Tampa, FL, USA). Measurements were taken by trained nurses, and all adolescents were required to sit and rest for at least 5 minutes before BP measurement. Participants were in a seated, relaxed position, with their feet resting flat on the ground. Two measurements were taken in the right arm, after a 5- and 10-minute rest. The mean of these 2 measurements was considered, but if the 2 measurements differed by $5 \mathrm{~mm} \mathrm{Hg}$ or more, a third measure was taken.

\subsection{Blood sampling}

After 10 hours of fasting, blood samples were collected from the antecubital vein between 8 and $10 \mathrm{AM}$, with participants in a sitting position. Blood samples were processed locally, then stored and shipped to a central laboratory where biochemical evaluations of all participants were performed. Serum glucose, triglyceride (TG), total cholesterol (TC), and high-density lipoprotein cholesterol (HDL-c) levels were determined by colorimetric methods using the Cobas Integra 400 Plus (ROCHE Diagnostics, Indianapolis, IN, USA). Fasting blood insulin was measured using chemiluminescence immunoassay (Immulite 2000; Diagnostic Products Corporation, Los Angeles, CA, USA). The ratio of TC to HDL-c was calculated as an 
index of atherogenic lipid profile. The homeostatic model assessment (HOMA), calculated as the product of basal glucose and insulin levels divided by 22.5 , was used as a proxy measure of insulin resistance [21].

\subsection{Pubertal stage}

To determine pubertal stage (which ranged from 1 to 5), each subject was asked to self-assess his/her development stage of secondary sex characteristics. Breast development in girls and genital development in boys were evaluated according to criteria outlined by Tanner and Whitehouse [22].

\subsection{Sociodemographic and lifestyle variables}

Adolescents answered a questionnaire that assessed several sociodemographic and lifestyle variables.

\subsubsection{Parental education}

For the present study, the highest level of parental education (measured by number of school years completed) was used as a proxy measure of socioeconomic status (SES). Participants were divided into 3 categories, reflecting divisions within the Portuguese educational system: mandatory or less $(\leq 9$ school years), secondary (10-12 school years), and college/university (>12 school years).

\subsection{Dietary intake}

Dietary intake was completed by adolescents and measured via a semiquantitative food frequency questionnaire (FFQ), validated for the Portuguese adults [23]. This semiquantitative FFQ was designed in accordance with criteria laid out by Willett [24] and adapted to include a variety of typical Portuguese food items. The FFQ was adapted for adolescents by including foods more frequently eaten by this age group [25]; the adolescent version covered the previous 12 months and comprised 91 food items or beverage categories. For each item, the questionnaire offered 9 frequency response options, ranging from "never" to "six or more times per day," and standard portion size and seasonality. Any foods not listed in the questionnaire could be listed by participants in a free-response section. Energy and nutritional intake were estimated with consideration of the respondents' ratings of frequency, portion, and seasonality of each item, using the software Food Processor Plus (ESHA Research Inc, Salem, OR, US). This program uses nutritional information from the United States that has been adapted for use with typical Portuguese foods and beverages. To verify the interitem consistency, the Cronbach $\alpha$ test was applied to the dimensions of the FFQ, and the score obtained $(\alpha=.892)$ was high, which indicated a good internal consistency.

For the present study, the "milk" group only included milk (whole, reduced-fat, and fat-free milk), the "yogurt" group included all types of yogurt, the "cheese" group included cottage and cream cheese, and the “total dairy" group included milk, yogurt, and cheese. Participants were divided into 2 categories, according to the amount of each food group consumed: one corresponding to an intake lower than or equal to the 
median amount of the total sample (low intake) and the other corresponding to an intake higher than the median amount of the total sample ("appropriate" intake).

\subsection{Cardiorespiratory fitness}

Cardiorespiratory fitness (CRF) was measured using the 20-m Shuttle-Run-Test, as previously described by Léger et al [26]. This test requires participants to run back and forth between 2 lines set $20 \mathrm{~m}$ apart. Running speed was set at $8.5 \mathrm{~km} / \mathrm{h}$ and increased by $0.5 \mathrm{~km} / \mathrm{h}$ each minute, reaching $18.0 \mathrm{~km} / \mathrm{h}$ at minute 20. A tape player announced the pace. Participants were told to keep up until exhausted. The test concluded for each participant either when the subject failed to reach the end lines concurrent with audio signals on 2 consecutive occasions or when the subject stopped because of fatigue. Participants were encouraged to keep running as long as possible. The number of shuttles performed by each participant was recorded. Adolescents were then classified into 2 groups, according to FITNESSGRAM-established age- and sexspecific cutoff points, as belonging to the healthy zone ("fit") or the unhealthy zone ("unfit") [27] and [28].

\subsection{Cardiometabolic risk score}

In this study, we computed a continuous cardiometabolic risk score (CMRS), according to Andersen et al [29], from the following measurements: TC/HDL-c ratio, TG, HOMA, systolic BP, percentage body fat, and CRF. For each one of these variables, age- and sex-adjusted $z$ scores were computed. The CMRS was constructed by summing the $z$ scores of all risk factors. Individuals with at least 1 SD were considered to be at high risk for cardiometabolic disease. As some authors suggested [29], the estimation of a CMRS that is based on the sum of age- and sex-adjusted $z$ scores for several metabolic risk factors constitutes a valid tool to use in the identification of children and adolescents at risk for developing cardiovascular disease and type 2 diabetes [30] and [31]. Indeed, the employment of such a score permits summation of all risk factors for an individual, creating an overall score that encompasses that person's cumulative risk and, at the same time and to some extent, accounts for the influence of growth and maturation on cardiovascular risk factors [30]. The CMRS computed for the purposes of this study only applies to this study population. A similar $z$ score approach was used previously in children and adolescents [31] and [32].

\subsection{Statistical analyses}

The Kolmogorov-Smirnov test was used to assess the assumption of normality. Weight, systolic BP, HDL$\mathrm{c}$, and TC/HDL-c ratio were logarithmically transformed to improve normality. An independent sample $t$ test or Mann-Whitney $U$ test was performed to compare continuous variables between CMRS groups, and the $\chi^{2}$ test was used with categorical variables. In this report, descriptive analysis is presented in terms of means and SDs, unless otherwise stated.

Separate multivariate logistic regression models were constructed to verify the relationship between high CMRS and dairy products intake, adjusting for parental education (reference - mandatory or less), pubertal 
stage, total energy intake (in kilocalories), protein intake (in percent of energy), total fat intake (in percent of energy), and dietary fiber (in grams per $1000 \mathrm{kcal}$ ). Dietary variables were entered as continuous variables. Furthermore, we adjusted the logistical model by underreporting energy intake, which was estimated using the ratio between reported energy intake and predicted basal metabolic rate [33] and [34]. The thresholds that defined low-energy reporters (underreporters) were 1.70 and 1.71 for girls and boys between 15 and 17 years old and 1.67 and 1.81 for girls and boys aged 18 years. "Low-energy reporter" (a categorical variable) was included in the model as a confounding factor.

Odds ratios (ORs) and 95\% confidence intervals (CIs) were computed for the appropriate dairy product intake categories. A $P$ value less than .05 was regarded as significant. All analyses were performed using PASW Statistic v.20 (SPSS, Chicago, IL, USA).

\section{Results}

The median amount of the total sample for total dairy, milk, yogurt, and cheese intake was 483.25 (454.35), 260.34 (400.94), 53.57 (116.66), and 12.86 (20.66) g/d, respectively.

Descriptive characteristics of the study sample within the context of dairy product intake are shown in Table 1. Adolescents with appropriate total dairy and milk intake had a higher proportion of parents with higher education level $(P=.002$, for both). Higher TG concentrations were seen in adolescents with appropriate cheese intake compared with those with low cheese intake $(63.5$ [36.0] mg/dL vs 60.0 [36.0] $\mathrm{mg} / \mathrm{dL}$, respectively). The higher proportion of unfit adolescents was seen in the low total dairy and milk intake group $(P<.05$, for both). In this study, all adolescents reported to be in Tanner $4(67.4 \%)$ or 5 (32.6\%; data not shown in Table 1). 


\begin{tabular}{|c|c|c|c|c|c|c|c|c|c|c|c|c|}
\hline & \multicolumn{2}{|c|}{ Total dairy } & \multirow[t]{2}{*}{ P } & \multicolumn{2}{|c|}{ Milk } & \multirow[t]{2}{*}{$P$} & \multicolumn{2}{|c|}{ Yogurt } & \multirow[t]{2}{*}{$P$} & \multicolumn{2}{|c|}{ Cheese } & \multirow[t]{2}{*}{$P$} \\
\hline & $\begin{array}{l}\text { Low } \\
\text { intake }\end{array}$ & $\begin{array}{l}\text { Appropriate } \\
\text { intake }\end{array}$ & & $\begin{array}{l}\text { Low } \\
\text { intake }\end{array}$ & $\begin{array}{l}\text { Appropriate } \\
\text { intake }\end{array}$ & & $\begin{array}{l}\text { Low } \\
\text { intake }\end{array}$ & $\begin{array}{l}\text { Appropriate } \\
\text { intake }\end{array}$ & & $\begin{array}{l}\text { Low } \\
\text { intake }\end{array}$ & $\begin{array}{l}\text { Appropriate } \\
\text { intake }\end{array}$ & \\
\hline Age $(y)^{a b}$ & $17.0(1.9)$ & $17.0(1.0)$ & .416 & $17.0(1.0)$ & $17.0(1.0)$ & .682 & $17.0(1.0)$ & $17.0(1.0)$ & .630 & $17.0(1.0)$ & $16.0(1.0)$ & .297 \\
\hline Height $(m)=x$ & $1.64(0.13)$ & $16.5(0.13)$ & .128 & $1.64(0.13)$ & $1.65(0.14)$ & 587 & $1.65(0.14)$ & $1.64(0.12)$ & .723 & $1.64(0.14)$ & $16.0(0.13)$ & .943 \\
\hline Weight $(\mathrm{kg})^{\text {cad }}$ & $63.2 \pm 12.9$ & $63.2 \pm 126$ & 958 & $63.7 \pm 13.4$ & $628 \pm 12.0$ & 518 & $63.0 \pm 12.1 \pm$ & $63.5 \pm 13.7$ & .864 & $63.2 \pm 126$ & $63.2 \pm 13.0$ & .965 \\
\hline Body fat $(\%)^{\text {cd }}$ & $21.4 \pm 8.5$ & $20.3 \pm 8.9$ & .114 & $21.4 \pm 8.6$ & $20.5 \pm 8.9$ & .242 & $20.8 \pm 8.3$ & $20.9 \pm 9.5$ & .800 & $20.6 \pm 8.4$ & $21.4 \pm 9.3$ & 336 \\
\hline $\begin{array}{l}\text { Waist } \\
\text { circumference }(\mathrm{cm})^{x b}\end{array}$ & $79.0(13.0)$ & $77.0(15.9)$ & .152 & $80.0(14.0)$ & $77.0(15.0)$ & .094 & $79.0(13.0)$ & $77.0(16.0)$ & .872 & $79.0(14.9)$ & $77.0(15.0)$ & .217 \\
\hline Systolic BP $(\mathrm{mm} \mathrm{H})^{\mathrm{cad}}$ & $116.3 \pm 15.8$ & $114.2 \pm 14.0$ & .135 & $116.6 \pm 15.6$ & $114.0 \pm 14.2$ & .068 & $114.5 \pm 15.3$ & $116.5 \pm 14.4$ & .155 & $115.1 \pm 15.0$ & $115.6 \pm 15.0$ & .708 \\
\hline Diastolic BP $\left(\mathrm{mm} \mathrm{H} \mathrm{H}_{2}\right)$ wb & $65.5(15.0)$ & $65.0(11.5)$ & 318 & $66.0(14.8)$ & $65.0(11.8)$ & $.46 B$ & 6.5 (12.5) & $66.5(13.8)$ & .534 & $65.0(12.9)$ & $66.5(11.6)$ & .922 \\
\hline $\mathrm{TC}(\mathrm{mg} / \mathrm{dL})^{\mathrm{c} d \mathrm{~d}}$ & $161.7 \pm 31.7$ & $1621 \pm 32.7$ & 942 & $163.1 \pm 33.9$ & $161.3 \pm 30.7$ & .534 & $161.9 \pm 321$ & $162.0 \pm 32.4$ & .923 & $160.1 \pm 30.7$ & $165.4 \pm 34.8$ & .071 \\
\hline HDL-c (mg/dL) $)^{c d}$ & $55.2 \pm 13.1$ & $55.3 \pm 13.4$ & 910 & $55.2 \pm 13.0$ & $55.5 \pm 13.0$ & .912 & $55.4 \pm 13.1$ & $55.0 \pm 13.4$ & .685 & $55.0 \pm 13.3$ & $55.8 \pm 13.1$ & .520 \\
\hline TC/HDL-e ratio ${ }^{c d}$ & $3.03 \pm 0.69$ & $3.06 \pm 0.82$ & 935 & $3.06 \pm 0.76$ & $3.03 \pm 0.76$ & .670 & $3.03 \pm 0.73$ & $3.07 \pm 0.81$ & .693 & $3.03 \pm 0.96$ & $3.08 \pm 0.79$ & 515 \\
\hline TGs (mg/dL) & $60.0(36.0)$ & $62.0(35.9)$ & 526 & $61.0(39.0)$ & $61.0(34.0)$ & 852 & $60.0(36.0)$ & $64.0(38.0)$ & .305 & $60.0(36.9)$ & $63.5(36.0)$ & .034 \\
\hline Fasting glucose $(\mathrm{mmol} / \mathrm{L})^{\mathrm{cad}}$ & $4.82 \pm 0.50$ & $4.82 \pm 0.54$ & 920 & $4.81 \pm 0.51$ & $4.82 \pm 0.53$ & 817 & $4.81 \pm 0.54$ & $4.83 \pm 0.48$ & .708 & $4.81=0.51$ & $4.83 \pm 0.54$ & .759 \\
\hline Fasting insulin $(\mu \mathrm{L} / \mathrm{mL}) \times b$ & $8.00(6.2)$ & $8.00(4.5)$ & .631 & $7.80(5.43)$ & $8.20(4.90)$ & .712 & $7.97(4.76)$ & $8.20(5.87)$ & .724 & $8.20(6.00)$ & $7.64(4.17)$ & .140 \\
\hline HOMA $A^{a b}$ & $1.71(1.31)$ & $1.73(1.03)$ & .6866 & $1.68(1.22)$ & $1.74(1.12)$ & .637 & $1.71(1.18)$ & $1.71(1.18)$ & .815 & $1.76(1.28)$ & $1.66(1.06)$ & .166 \\
\hline CMRS (z score) c,d & $0.034 \pm 3.130$ & $0.025 \pm 2800$ & 895 & $0.077 \pm 3.161$ & $0.009 \pm 2.745$ & .797 & $-0.059 \pm 2.920$ & $0.176 \pm 3.040$ & .430 & $-0.016 \pm 2924$ & $0.119=3.050$ & .614 \\
\hline \multicolumn{13}{|l|}{ Weight status $(\%)^{*}$} \\
\hline Obese & 8.5 & 5.7 & & 8.8 & 5.3 & & 6.2 & 86 & & 8.0 & 5.4 & \\
\hline \multicolumn{13}{|l|}{$\operatorname{sex}(\%)^{*}$} \\
\hline Boys & 38.5 & 45.7 & .101 & 40.2 & 44.1 & 377 & 42.3 & 41.7 & .90 & 41.3 & 43.7 & .605 \\
\hline Girls & 61.5 & 54.3 & & 59.8 & 55.9 & & 57.7 & 58.3 & & 58.7 & 56.3 & \\
\hline \multicolumn{13}{|l|}{ Parental education (\%) } \\
\hline Mandatory or less & 67.2 & 53.0 & .000 & 67.1 & 53.1 & .002 & 61.9 & 57.2 & .193 & 61.8 & 56.9 & .482 \\
\hline Secondary & 23.9 & 29.1 & & 24.1 & 29.0 & & 23.8 & 31.0 & & 26.0 & 27.5 & \\
\hline College/ & 8.9 & 17.8 & & 8.8 & 180 & & 14.3 & 11.8 & & 12.2 & 15.6 & \\
\hline \multicolumn{13}{|l|}{ University } \\
\hline \multicolumn{13}{|l|}{$\operatorname{CRF}(\%)^{*}$} \\
\hline Unfit & 66.4 & 51.8 & .001 & 65.9 & 522 & .002 & 57.7 & 61.5 & .399 & 59.6 & 58.1 & .740 \\
\hline Fit & 33.6 & 48.2 & & 34.1 & 47.8 & & 42.3 & 38.5 & & 40.4 & 41.9 & \\
\hline
\end{tabular}

The energy intakes and dietary characteristics of the study sample, according to dairy product intake, are presented in Table 2. Adolescents with appropriate dairy product intake (total, milk, yogurt, and cheese) had higher total energy intake than did adolescents with low dairy product intake $(P<.001$, for all). Higher protein intake was seen in adolescents with appropriate total dairy and milk intake $(P<.001$, for all). Adolescents with appropriate yogurt intake had higher carbohydrate and dietary fiber intake and lower total fat intake compared with adolescents with low yogurt intake $(P<.05$, for all). However, adolescents with appropriate cheese intake had lower carbohydrate and higher total fat intake than did adolescents with low cheese intake $(P<.05$, for all). 


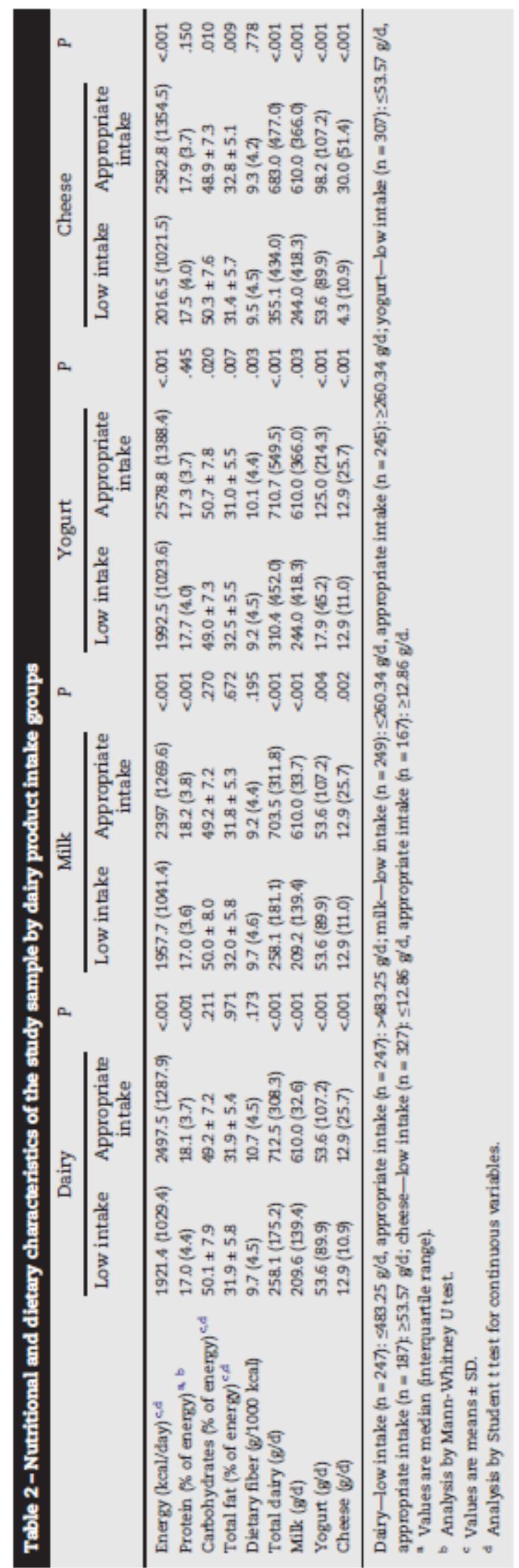

As indicated in Fig., adolescents with appropriate milk intake had lower CMRS than did adolescents with low milk intake $(10.6 \%$ vs $18.1 \%, P=.026)$. There was no significant difference between these groups regarding total dairy $(11.3 \%$ vs $17.4 \%, P=.054)$, yogurt $(14.4 \%$ vs $14.3 \%, P=.974)$, and cheese intake (16.2\% vs $13.5 \%, P=.416)$. 


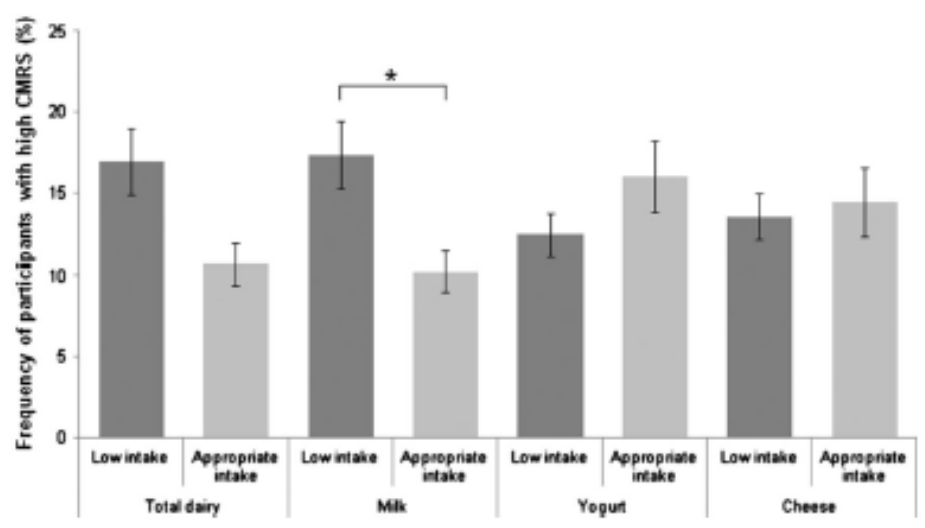

Fig - Proportion of adolescents with high CMRS across the different dairy product groups. $\mathrm{P}=.026$, for comparis on between low milk and appropriate milk intake. Dairy-low intake: $\leq 483.25 \mathrm{~g} / \mathrm{d}$, appropriate intake: $>483.25 \mathrm{~g} / \mathrm{d} ;$ milk-low intake: $\leq 260.34 \mathrm{~g} /$ $\mathrm{d}$, appropriate intake: $\geq 260.34 \mathrm{~g} / \mathrm{d} ;$ yogurt - low intake: $\leq 53.57 \mathrm{~g} / \mathrm{d}$, appropriate intake: $\geq 53.57 \mathrm{~g} / \mathrm{d}$; cheese-low intake: $\leq 1286 \mathrm{~g} /$ $\mathrm{d}$, appropriate intake: $\geq 1286 \mathrm{~g} / \mathrm{d}$. Values are means $\pm \mathrm{SD}$.

The results of separate multivariate logistic regression analysis, predicting high CMRS from dairy product intake, are shown in Table 3. After adjusting for demographic and dietary variables, adolescents with appropriate milk intake were less likely to have high CMRS than those with low milk intake (OR, 0.531; $95 \% \mathrm{Cl}, 0.302-0.931)$. To control the possible confounding of yogurt and cheese intake, we performed an additional milk multivariate model analysis where we adjusted for these 2 variables, and similar results were observed (OR, 0.506; 95\% $\mathrm{Cl}, 0.287-0.894)$.

\begin{tabular}{|c|c|c|c|c|}
\hline & \multicolumn{2}{|c|}{ Model 1} & \multicolumn{2}{|c|}{ Model 2} \\
\hline & OR $(95 \%$ CI) & P & OR $(95 \%$ CI) & $P$ \\
\hline \multicolumn{5}{|c|}{ Total dairy intake } \\
\hline Low & Reference & & Reference & \\
\hline Appropriate & $0.607(0.363-1.013)$ & .056 & $0.621(0.355-1.085)$ & .094 \\
\hline \multicolumn{5}{|l|}{ Milk intake } \\
\hline Low & Reference & & Reference & \\
\hline Appropriate & $0.538(0.320-0.904)$ & .019 & $0.531(0.302-0.931)$ & .027 \\
\hline \multicolumn{5}{|l|}{ Yogurt intake } \\
\hline low & Reference & & Reference & \\
\hline Appropriate & $1.009(0.601-1.693)$ & .974 & $0.707(0.636-1.948)$ & .707 \\
\hline \multicolumn{5}{|l|}{ Cheese intake } \\
\hline Low & Reference & & Reference & \\
\hline Appropriate & $1.240(0.737-2.087)$ & .417 & $1.533(0.875-2.656)$ & .135 \\
\hline
\end{tabular}

Dairy-low intake $(n=247): 5483.25 \mathrm{~g} / \mathrm{d}$, appropriate intake $(\mathrm{n}=247): 2.83 .25 \mathrm{~g} / \mathrm{d}$; milk-low intake $(\mathrm{n}=249): \leq 260.34 \mathrm{~g} / \mathrm{d}$, appropriate intake $(\mathrm{n}=$ 245): $3260.34 \mathrm{~g} / \mathrm{d}$; yogurt-low intake $(\mathrm{n}=307): 533.57 \mathrm{~g} / \mathrm{d}$, appropriate intake $(\mathrm{n}=187): 353.57 \mathrm{~g} / \mathrm{d}$; cheese-low intake $(\mathrm{n}=327): \leq 12.86 \mathrm{~g} / \mathrm{d}$, appropriate intake $(\mathrm{n}=167): \geq 12.86 \mathrm{~g} / \mathrm{d}$. Model 1 : unadjusted model; model 2: adjusted for parental education (reference: mandatory or less), pubertal stage, bwenergy reporter, energy intake (in kibcalories), total fat intake (percent of energy), protein intake (percent of energy), and dietary fiber intake (in grams per $1000 \mathrm{kcal}$ ).

\section{Discussion}

The present study explored the association between dairy product intake and the clustering of cardiometabolic risk factors in adolescents. The results indicated that adolescents holding higher milk intake were less likely to have high CMRS than those who had lower milk intake. This association was not 
confounded by other lifestyle factors or nutritional variables because it remained significant after adjustment.

Previous cross-sectional [11] and [14] and prospective [15] and [16] studies show an inverse relationship between milk or dairy product intake and cardiometabolic risk factors, in children and adolescents. It seems that milk and/or dairy product intake reduces the risk of central obesity [11] and [14], high BP [15] and [16], and insulin resistance [14]. However, studies examining the association between dairy product intake and clustering of cardiometabolic risk factors are limited in the pediatric population. Kelishadi et al, [35] in an Iranian youth cross-sectional study, found that an age-adjusted OR for having cardiometabolic disease (the metabolic syndrome [MetS]) lowered significantly as dairy consumption (milk, yogurt, and cheese) increased in frequency. However, in their analysis, this association was not explored with different types of dairy products, as were the associations in our study.

A number of observational studies have examined the association between dairy product intake and cardiometabolic risk among adults. Azadbakht et al [1] showed that subjects in the highest quartile of dairy consumption (milk, yogurt, cheese, and milk-based-desserts) had lower odds of having enlarged waist circumference, hypertension, and MetS. Likewise, in the Coronary Artery Risk Development in Young Adults study, Pereira et al [8] found that among individuals (aged 18-30 years) who were overweight at baseline, dairy consumption was inversely associated with the incidence of obesity, abnormal glucose homeostasis, dyslipidemia, and hypertension over the next 10 years. In contrast, findings from the Hoorn cohort found that higher dairy product intake was significantly associated with higher fasting glucose concentrations and lower diastolic BP, but not with body weight or other metabolic variables (ie, lipids, postload glucose, or insulin [36]. In the latter study, when different dairy products were distinguished, significant inverse associations were observed for dairy desserts, milk, and yogurt with systolic and diastolic BP, whereas cheese consumption was positively associated with BMI. Likewise, in our study, when all types of dairy products were pooled together, no significant association was found with CMRS. Thus, the findings of this study also reveal the need to disaggregate dairy products in analyses that explore the relationship of these variables with cardiometabolic outcomes. Given that dairy processing generates a variety of biochemical changes to milk composition, including the loss of more labile constituents (eg, vitamin $\mathrm{C}$ and enzymes), the removal of bioactive components (eg, whey removal from cheese), and/or the addition of ingredients (eg, the addition of sugar to yogurt and salt to cheese) [37]. Thus, the use of total dairy intake may not reveal the underlying relationships between milk and cardiometabolic risk in adolescents. Furthermore, the lack of association observed between CMRS and cheese may be, in part, explained by the higher fat, sodium, and lower potassium held by cheese compared with milk and other fluid dairy food [38]. On the other hand, the preponderance of evidence, mostly in adults, indicates that milk intake is more likely to be associated with beneficial cardiometabolic outcomes [13], [39], [40] and [41]. Few studies have shown the beneficial effects of other dairy products [10], [36] and [42]. 
Despite the existence of discrepancies, most epidemiologic studies do suggest that dairy products may play a role in the prevention of cardiometabolic risk factors [7]. However, it is unclear which dairy product components may explain their preventive effects, and the mechanisms behind these effects are not well understood. Current evidence indicates that the beneficial effects of dairy products may be attributable to milk fat, calcium, magnesium, potassium, bioactive peptides, or the combination of these, as part of the unique nutrient package that dairy provides [43]. Calcium may affect cardiometabolic risk factors by regulating energy metabolism via the reduction of lipogenesis and enhancement of lipolysis in adipocyte, increasing both fecal fat excretion and fat oxidation. It has been suggested that calcium, as well as magnesium and potassium, controls BP through multiple mechanisms, such as modulating intracellular calcium concentration, decreasing sodium retention, inhibiting proinflammatory events in vascular smooth muscle cells, reducing platelet aggregation, and decreasing renal vascular resistance [43] and [44]. Furthermore, the temperate Atlantic climate in the Azores Islands allows milk production, which is heavily based on grazing, almost all year round [45]. It has long been described that the milk fat produced from the pasture is rich in unsaturated fatty acids, including trans-octadecenoic acids and conjugated isomers of linoleic acid [45] and [46]. The conjugated isomers of linoleic acid has been associated with improving dyslipidemia and the proinflammatory state related to obesity and the MetS [47], [48] and [49]. Emerging scientific findings also indicate that such milk fat components as butyric acid may favor fat oxidation and decrease glycogen use [50]. Moreover, it seems that bioactive peptides derived from milk proteins may act independently of calcium to prevent the development of cardiometabolic risk factors and its components via a wide range of mechanisms that include the satiety response, the regulation of insulinemia levels and BP, the uptake of free radicals, and the alteration of lipid profiles [13].

It should be noted that the inverse association between milk intake and CMRS may also be attributed to the overall healthier lifestyle associated with higher milk intake. For instance, in our study, a higher proportion of fit adolescents was seen in high total dairy and milk intake groups. These findings are in line with the results of the Healthy Lifestyle in Europe by Nutrition in Adolescence study that found a positive association between CRF and dairy product intake among adolescents [51].

In addition to possibly exerting beneficial effects on cardiometabolic risk factors, milk is an excellent nutrient source for adolescents, who experience a period characterized by dynamic changes that occur in response to growth and puberty. Several studies have demonstrated that when milk is largely excluded from an adolescent's diet, it is difficult for him to achieve the daily requirements for calcium and other nutrients [52], [53] and [54]. Hence, consumption of the recommended amounts of milk may help adolescents meet their nutritional requirements and improve their diet quality [55] and [56]. It is important to note that the median amount reported in the appropriate categories of dairy consumption is consistent with current Portuguese recommendations for 2 to 3 servings of dairy products per day as part of a healthy diet [57]. According to a study with a representative sample of the Portuguese population, milk is consumed in higher proportions (63\%) than other dairy products (yogurt and cheese), and the Azorean Islands is a region where the proportion of milk consumption is higher compared with the other Portuguese regions [58] and [59]. 
In the present study, adolescents with parents holding higher education levels seemed to present higher intakes of total dairy and milk. According to evidence, it appears that milk and dairy consumption might be a marker of SES, with lower intake seen in lower-SES households [60], [61] and [62]. It has been postulated that the rise of healthy food consumption seen in higher SES households may be caused by the knowledge and health awareness or the increased pressures exerted to attain social acceptability, which occurs when SES increases [60]. Parental health awareness may help children and adolescents develop strategies for choosing healthy foods at home and in social situations. In addition, previous findings of the same cohort indicated that SES was a positive obesity predictor [63].

Some limitations of our study should be addressed. First, it should be noted that, as in other cross-sectional studies, conclusions related to cause and effect cannot be drawn. Second, although we did not separate high- and low-fat milk, we controlled for fat intake effects on our data. Moreover, although all types of milk were consumed by adolescents, in our sample, most participants $(92.7 \%)$ had consumed semiskimmed $(84.3 \%)$ and skimmed milk (8.4\%). In addition, it has been established that milk fat, despite containing saturated fat, has beneficial effects on serum lipids [64] and is not associated with a higher incidence of cardiovascular disease [65] and [66] or cardiometabolic risk factors [43], [66] and [67]. Finally, with the use of self-reported dietary intake data, one cannot rule out some reporting bias. However, the FFQ has been previously tested [68] and [69], and the analysis was controlled to prevent the misreporting of energy intake. Another FFQ limitation, as in other self-administered questionnaires, is the reliance on participants' recall and the recruitment of motivated participants [24] and [70]. However, adolescents were given oral instructions and had completed it with the help of the physical education teachers specially trained for the data collection, which may have improved the quality of information.

The strengths of this study include its adjustment for a variety of important confounders such as pubertal stage and dietary variables, as well as the fact that it was conducted with adolescents. This is a population about whom little is known with regard to the associations between dairy intake and the clustering of cardiometabolic risk factors.

In summary, our results do not support the initial hypothesis that higher dairy intake is associated with lower cardiometabolic risk factors clustering in adolescents, given that only milk intake was negatively associated with lower cardiometabolic risk factors clustering in adolescents. Evidence from prospective and randomized clinical investigations is needed to examine the effects of long-term consumption of milk and dairy products on cardiometabolic risk factors. Our findings may also encourage further research on the effects that different types of dairy products have on cardiometabolic risk factors in adolescents.

\section{References}


[1]L. Azadbakht, et al.Dairy consumption is inversely associated with the prevalence of the metabolic syndrome in Tehranian adults Am J Clin Nutr, 82 (2005), pp. 523-530

[2]S.M. Camhi, P.T. Katzmarzyk Tracking of cardiometabolic risk factor clustering from childhood to adulthood Int J Pediatr Obes, 5 (2010), pp. 122-129

[3]A.M. Tailor, et al. An update on the prevalence of the metabolic syndrome in children and adolescents Int J Pediatr Obes, 5 (2010), pp. 202-213

[4] J.A. Morrison, et al. Metabolic syndrome in childhood predicts adult metabolic syndrome and type 2 diabetes mellitus 25 to 30 years later J Pediatr, 152 (2008), pp. 201-206

[5] L.B. Andersen, et al. Biological cardiovascular risk factors cluster in Danish children and adolescents: the European Youth Heart Study Prev Med, 37 (2003), pp. 363-367

[6] S.M. Grundy, et al. Diagnosis and management of the metabolic syndrome. An American Heart Association/National Heart, Lung, and Blood Institute Scientific Statement. Executive summary Cardiol Rev, 13 (2005), pp. 322-327

[7] L.E. van Meijl, R. Vrolix, R.P. Mensink Dairy product consumption and the metabolic syndrome Nutr Res Rev, 21 (2008), pp. 148-157

[8] M.A. Pereira, et al. Dairy consumption, obesity, and the insulin resistance syndrome in young adults: the CARDIA Study JAMA, 287 (2002), pp. 2081-2089

[9] M.M. McGrane, et al. Dairy consumption, blood pressure, and risk of hypertension: an evidence-based review of recent literature Curr Cardiovasc Risk Rep, 5 (2011), pp. 287-298

[10] A.C. Vergnaud, et al. Dairy consumption and 6-y changes in body weight and waist circumference in middle-aged French adults Am J Clin Nutr, 88 (2008), pp. 1248-1255

[11] M.L. Bradlee, et al. Food group intake and central obesity among children and adolescents in the Third National Health and Nutrition Examination Survey (NHANES III) Public Health Nutr, 13 (2010), pp. 797-805

[12] E. Wirfalt, et al. Food patterns and components of the metabolic syndrome in men and women: a cross-sectional study within the Malmo Diet and Cancer cohort Am J Epidemiol, 154 (2001), pp. 1150-1159

[13] I. Ricci-Cabello, M.O. Herrera, R. Artacho Possible role of milk-derived bioactive peptides in the treatment and prevention of metabolic syndrome Nutr Rev, 70 (2012), pp. 241-255

[14] V. Hirschler, et al. Inverse association between insulin resistance and frequency of milk consumption in low-income Argentinean school children J Pediatr, 154 (2009), pp. 101-105

[15] L.L. Moore, et al. Intake of fruits, vegetables, and dairy products in early childhood and subsequent blood pressure changeEpidemiology, 16 (2005), pp. 4-11

[16] A.M. Rangan, et al. The effect of dairy consumption on blood pressure in mid-childhood: CAPS cohort study Eur J Clin Nutr, 66 (2012), pp. 652-657

[17] Instituto Nacional Estatística Estimativas da População Residente, segundo Grandes Grupos Etários e Sexo (2003) [[cited 2008 November]; Available at: http://www.ine.pt/] 
[18] World Health Organization Measuring obesity: classification and description of anthropometric data Report on a WHO Consultation on the Epidemiology of Obesity, World Health Organization, Regional Office for Europe, Copenhagen (1988)

[19] T.J. Cole, et al. Establishing a standard definition for child overweight and obesity worldwide: international survey BMJ, 320 (2000), pp. 1240-1243

[20] T.J. Cole, et al. Body mass index cut offs to define thinness in children and adolescents: international survey BMJ, 335 (2007), p. 194

[21] D.R. Matthews, et al. Homeostasis model assessment: insulin resistance and beta-cell function from fasting plasma glucose and insulin concentrations in man Diabetologia, 28 (1985), pp. 412-419

[22] J.M. Tanner, R.H. Whitehouse Clinical longitudinal standards for height, weight, height velocity, weight velocity, and stages of puberty Arch Dis Child, 51 (1976), pp. 170-179

[23] C. Lopes, et al. Intake and adipose tissue composition of fatty acids and risk of myocardial infarction in a male Portuguese community sample J Am Diet Assoc, 107 (2007), pp. 276-286

[24] W. Willett Food frequency methods in nutritional epidemiology, Oxford University Press, New York (1998), pp. 74-100

[25] D. Silva, C. Rego, A. Guerra Characterization of food habits and comparative study between two methods of food assessment in adolescents Rev Aliment Humana, 10 (2004), pp. 33-40

[26] L.A. Léger, et al. The multistage 20 metre shuttle run test for aerobic fitness

J Sports Sci, 6 (1988), pp. 93-101

[27] K.J. Cureton, G.L. Warren Criterion-referenced standards for youth health-related fitness tests: a tutorial Res Q Exerc Sport, 61 (1990), pp. 7-19

[28] The Cooper Institute for Aerobics Research FITNESSGRAM test administration manual

(3rd ed.)Human Kinetics, Champaign (IL) (2004)

[29] L.B. Andersen, et al. Physical activity and clustered cardiovascular risk in children: a crosssectional study (The European Youth Heart Study) Lancet, 368 (2006), pp. 299-304

[30] F. Lobelo, et al. Cardiorespiratory fitness and clustered cardiovascular disease risk in U.S. adolescents J Adolesc Health, 47 (2010), pp. 352-359

[31] G.L. Ambrosini, et al. Dietary patterns and markers for the metabolic syndrome in Australian adolescents Nutr Metab Cardiovasc Dis, 20 (2010), pp. 274 - 283

[32] C. Moreira, et al. Cardiorespiratory fitness is negatively associated with metabolic risk factors independently of the adherence to a healthy dietary pattern Nutr Metab Cardiovasc Dis, 23 (7) (2012), pp. 670-676

[33] G.R. Goldberg, et al. Critical evaluation of energy intake data using fundamental principles of energy physiology: 1 . Derivation of cut-off limits to identify under-recording

[34] A.E. Black Critical evaluation of energy intake using the Goldberg cut-off for energy intake:basal metabolic rate. A practical guide to its calculation, use and limitations Int J Obes Relat Metab Disord, 24 (2000), pp. 1119-1130 
[35] R. Kelishadi, et al. Factors associated with the metabolic syndrome in a national sample of youths: CASPIAN Study Nutr Metab Cardiovasc Dis, 18 (2008), pp. 461-470

[36] M.B. Snijder, et al. Is higher dairy consumption associated with lower body weight and fewer metabolic disturbances? The Hoorn Study Am J Clin Nutr, 85 (2007), pp. 989-995

[37] A.S. Wiley Dairy and milk consumption and child growth: is BMI involved? An analysis of NHANES 1999-2004 Am J Hum Biol, 22 (2010), pp. 517-525

[38] R.A. Ralston, et al. A systematic review and meta-analysis of elevated blood pressure and consumption of dairy foods J Hum Hypertens, 26 (2012), pp. 3-13

[39] P.C. Elwood, J.E. Pickering, A.M. Fehily Milk and dairy consumption, diabetes and the metabolic syndrome: the Caerphilly prospective study J Epidemiol Community Health, 61 (2007), pp. 695-698

[40] G. Samuelson, et al. Dietary fat intake in healthy adolescents: inverse relationships between the estimated intake of saturated fatty acids and serum cholesterol Br J Nutr, 85 (2001), pp. 333-341

[41] M.P. St-Onge, L.L. Goree, B. Gower High-milk supplementation with healthy diet counseling does not affect weight loss but ameliorates insulin action compared with low-milk supplementation in overweight children J Nutr, 139 (2009), pp. 933-938

[42] M.A. Beydoun, et al. Ethnic differences in dairy and related nutrient consumption among US adults and their association with obesity, central obesity, and the metabolic syndrome Am J Clin Nutr, 87 (2008), pp. 1914-1925

[43] B.H. Rice, et al. Dairy components and risk factors for cardiometabolic syndrome: recent evidence and opportunities for future research Adv Nutr, 2 (2011), pp. 396-407

[44] P.M. Kris-Etherton, et al. Milk products, dietary patterns and blood pressure management J Am Coll Nutr, 28 (2009), pp. 103S-119S

[45] O.A. Rego, et al. Effect of diet on the fatty acid pattern of milk from dairy cows Anim Res, 53 (2004), pp. $213-220$

[46] R.R. Riel Physico-chemical characteristics of Canadian milk fat-unsaturated fatty acids

J Dairy Sci, 46 (1963), p. 102

[47] R.S. McLeod, et al. Conjugated linoleic acids, atherosclerosis, and hepatic very-low-density lipoprotein metabolism Am J Clin Nutr, 79 (2004), pp. 1169S-1174S

[48] M.M. Jacome-Sosa, et al. Increased hypolipidemic benefits of cis-9, trans-11 conjugated linoleic acid in combination with trans- 11 vaccenic acid in a rodent model of the metabolic syndrome, the JCR: LA-cp rat Nutr Metab (2010), p. 7

[49] C.M. Reynolds, H.M. Roche Conjugated linoleic acid and inflammatory cell signalling Prostaglandins Leukot Essent Fat Acids, 82 (2010), pp. 199-204

[50] C.P. Marinangeli, P.J. Jones Pulse grain consumption and obesity: effects on energy expenditure, substrate oxidation, body composition, fat deposition and satiety $\mathrm{Br} J \mathrm{Nutr}, 108$ (2012), pp. S46-S51 
[51] M. Cuenca-Garcia, et al. Cardiorespiratory fitness and dietary intake in European adolescents: the Healthy Lifestyle in Europe by Nutrition in Adolescence study Br J Nutr, 107 (2012), pp. 1850-1859

[52] X. Gao, et al. Meeting adequate intake for dietary calcium without dairy foods in adolescents aged 9 to 18 years (National Health and Nutrition Examination Survey 2001-2002) J Am Diet Assoc, 106 (2006), pp. 1759-1765

[53] S.L. Goolsby, et al. Consumption of calcium among African American adolescent girls Ethn Dis, 16 (2006), pp. 476-482

[54] C.M. Weaver Role of dairy beverages in the diet Physiol Behav, 100 (2010), pp. 63-66

[55] L.A. Spence, C.J. Cifelli, G.D. Miller The role of dairy products in healthy weight and body composition in children and adolescents Curr Nutr Food Sci, 7 (2011), pp. 40-49

[56] T.A. Marshall, et al. Diet quality in young children is influenced by beverage consumption J Am Coll Nutr, 24 (2005), pp. 65-75

[57] S.S. Rodrigues, et al. A new food guide for the Portuguese population: development and technical considerations J Nutr Educ Behav, 38 (2006), pp. 189-195

[58] Sociedade Portuguesa de Ciências da Nutrição e Alimentação Alimentação e Estilos de Vida da População Portuguesa: Como comem os portugueses? 8o Congresso da Sociedade Portuguesa de Ciências da Nutrição e Alimentação, Sociedade Portuguesa de Ciências da Nutrição e Alimentação, Porto (2009)

[59] R.I. Poínhos, et al. Alimentação e estilos de vida da população portuguesa: metodologia e resultados preliminares Revista da Sociedade Portuguesa de Ciências da Nutrição e Alimentação, 15 (2009), pp. 43-60

[60] P. Deshmukh-Taskar, et al. Does food group consumption vary by differences in socioeconomic, demographic, and lifestyle factors in young adults? The Bogalusa Heart Study J Am Diet Assoc, 107 (2007), pp. 223-234

[61] N.I. Larson, et al. Calcium and dairy intakes of adolescents are associated with their home environment, taste preferences, personal health beliefs, and meal patterns J Am Diet Assoc, 106 (2006), pp. 1816-1824

[62] D. Neumark-Sztainer, et al. Correlates of inadequate consumption of dairy products among adolescents J Nutr Educ Behav, 29 (1997), pp. 12-20

[63] S. Abreu, et al. Food consumption, physical activity and socio-economic status related to BMI, waist circumference and waist-to-height ratio in adolescentsPublic Health Nutr (2013), pp. $1-16$

[64] R.P. Mensink, et al.

Effects of dietary fatty acids and carbohydrates on the ratio of serum total to HDL cholesterol and on serum lipids and apolipoproteins: a meta-analysis of 60 controlled trials Am J Clin Nutr, 77 (2003), pp. 1146-1155

[65] J.B. German, et al. A reappraisal of the impact of dairy foods and milk fat on cardiovascular disease risk Eur J Nutr, 48 (2009), pp. 191-203 
[66] M. Pfeuffer, J. Schrezenmeir Milk and the metabolic syndrome Obes Rev, 8 (2007), pp. 109118

[67] P.C. Elwood, et al. The consumption of milk and dairy foods and the incidence of vascular disease and diabetes: an overview of the evidence Lipids, 45 (2010), pp. 925-939

[68] E. Ramos Health determinants in Porto adolescents-the Epiteen cohort University of Porto, Porto (2006)

[69] J. Mota, C. Esculcas Leisure-time physical activity behavior: structured and unstructured choices according to sex, age, and level of physical activity Int J Behav Med, 9 (2002), pp. 111121

[70] F. Lodato, et al. Caffeine intake reduces sleep duration in adolescents Nutr Res, 33 (2013), pp. $726-732$ 